\title{
Malformaciones de la vena cava inferior y tromboembolismo venoso
}

\author{
Inferior vena cava malformations and venous \\ thromboembolism
}

\author{
Lucía Beatriz Taboada, Ana Cristina Montenegro, Bibiana Pinzón, \\ Jaime Andrés Alvarado • Bogotá, D.C. (Colombia)
}

\section{Resumen}

Presentamos el caso de una paciente de 30 años con tromboembolismo pulmonar de riesgo alto por PESI, en quien se confirmó la presencia de una anomalía congénita de la vena cava inferior, dada por ausencia de su porción intrahepática y subsecuente drenaje por el sistema ácigos. Como factor de riesgo adicional coexistía el consumo de anticonceptivos orales.

Las malformaciones congénitas de la vena cava inferior constituyen una entidad poco frecuente y la mayoría cursan de forma indolente identificándose como hallazgo incidental. Pueden considerarse como factor de riesgo para tromboembolismo venoso especialmente en pacientes menores de 30 años con trombosis venosa profunda de miembros inferiores con compromiso de las venas iliacas, cuando hay resistencia a anticoagulantes o trombosis venosa profunda recurrente. No existe una recomendación clara respecto a la duración de la anticoagulación por lo que se debe individualizar cada caso teniendo en cuenta el perfil de riesgo de cada paciente. (Acta Med Colomb 2015; 40: 54-57).

Palabras clave: vena cava inferior, anomalías congénitas, trombosis venosa,embolia pulmonar, anticoagulantes.

\section{Abstract}

The case of a 30 year old female patient with high-risk pulmonary embolism by PESI, in whom the presence of a congenital anomaly of the inferior vena cava due to the absence of its intrahepatic portion and subsequent drainage through the azygos system was confirmed, is presented. An additional risk factor was the consumption of oral contraceptives..

Congenital malformations of the inferior vena cava are a rare entity and most follow an indolent course being identified as an incidental finding. They can be considered as a risk factor for venous thromboembolism especially in patients younger than 30 years with deep venous thrombosis of lower limbs with involvement of the iliac veins, when there is resistance to anticoagulants or recurrent deep vein thrombosis. There is no clear recommendation regarding the duration of anticoagulation so each case must be individualized taking into account the risk profile of each patient. (Acta Med Colomb 2015; 40: 54-57).

Keywords: inferior vena cava, congenital abnormalities, venous thrombosis, pulmonary embolism, anticoagulants.
Dra. Lucía Beatriz Taboada: Epidemióloga Clínica, Residente de Medicina Interna; Dra. Ana Cristina Montenegro: Especialista en Medicina Interna, Clínica de Anticoagulación; Dra. Bibiana Pinzón: Radióloga; Dr. Jaime Andrés Alvarado: Especialista en Medicina Interna y Neumología. Hospital Universitario Fundación Santa Fe de Bogotá. Bogotá, D.C. (Colombia). Correspondencia. Dr. Jaime Andrés Alvarado. Bogotá, D.C. (Colombia).

Correo electrónico: jaimeaalvarado@gmail.com Recibido: 10/III/2014 Aceptado: 23/II/2015

\section{Introducción}

Las malformaciones de la vena cava inferior constituyen una entidad rara con una prevalencia de $0.3-0.5 \%$ en la población general y hasta de $2 \%$ en población con anomalías cardiovasculares (1). Al favorecer la estasis venosa, constituyen un factor de riesgo para el tromboembolismo venoso, en especial cuando se asocian a otros factores de riesgo $(2,3)$. Las recomendaciones con respecto al tipo y duración de la anticoagulación deben ser individualizadas. A continuación se presenta un caso clínico y se discuten las implicaciones para el diagnóstico y el manejo.

\section{Presentación del caso}

Paciente femenina de 30 años, con cuadro de disnea súbita hasta mMRC 4 que inició durante la marcha, asociada a palpitaciones y dolor torácico retroesternal y subcostal 
derecho de moderada intensidad, sin irradiación ni factores desencadenantes y que no mejoraba con el reposo. En los últimos 15 días previos al ingreso había presentado disnea que pasó de mMRC 1 a mMRC 2, dolor pleurítico bibasal $\mathrm{y}$ tos seca ocasionalmente con pintas hemoptoicas. No se evidencian en el interrogatorio antecedentes familiares ni personales de importancia, excepto por el consumo de anticonceptivos orales.

A su ingreso a urgencias se encuentra con tensión arterial limítrofe y taquicardia, siendo evidente marcada dificultad respiratoria dada por polipnea, uso de músculos accesorios y desaturación al ambiente. Al examen físico se encuentra palidez mucocutánea, ruidos cardiacos rítmicos taquicárdicos, disminución de ruidos respiratorios en las bases y ausencia de signos de trombosis venosa profunda.

Se deja como impresión diagnóstica tromboembolismo pulmonar de alta probabilidad (puntaje de Wells 5.5 en clasificación bimodal) (4) y se inicia manejo en observación de urgencias con oxígeno y anticoagulación con heparina de bajo peso molecular. Se toman paraclínicos que muestran: hemograma sin anemia ni alteraciones en las plaquetas; gases arteriales con alcalemia respiratoria y PAFI de 217; tiempos de coagulación normales y electrocardiograma con taquicardia sinusal y patrón S1Q3T3. La tomografía de tórax con protocolo para tromboembolismo pulmonar muestra extenso compromiso embólico que compromete arteria pulmonar principal, con signos de repercusión hemodinámica e infarto pulmonar en lóbulo inferior izquierdo y probable interrupción de la vena cava inferior con continuidad a través del sistema ácigos como variante. El doppler venoso de miembros inferiores fue negativo para trombosis venosa profunda.

Se procede a realizar estratificación de riesgo, encontrándose troponina positiva y ecocardiograma transtorácico con septum interventricular aplanado con movimiento paradójico, dilatación moderada del ventrículo derecho e hipoquinesia severa de la pared anterior con presión sistólica de la arteria pulmonar de $48 \mathrm{mmHg}$.

Teniendo en cuenta el alto riesgo de deterioro y falla ventilatoria en el contexto de una paciente joven con puntaje de PESI (5) en 118 y con los paraclínicos ya descritos, se decide traslado a Unidad de Cuidados Intensivos. Permanece estable hemodinámicamente, sin embargo por el riesgo aumentado de progresión a soportes invasivos y ante la posiblidad elevada de hipertensión pulmonar residual en el futuro, se considera que el perfil de riesgo beneficio de la trombolisis es bueno, por lo que se inicia activador tisular del plasminógeno humano recombinante (rTPA) en esquema corto. La paciente evoluciona favorablemente con mejoría en variables hemodinámicas, oxigenación y normalización de los signos de falla derecha aguda en el ecocardiograma. Como única complicación hemorrágica presenta hematoma en brazo derecho que no requiere intervención.

Se decide anticoagulación a largo plazo con warfarina con meta de INR entre 2 y 3 . Se complementa estudio con tomografía de abdomen que confirma la presencia de anomalía congénita de la vena cava inferior por ausencia de su porción intrahepática y drenaje por el sistema ácigos (Figura 1).
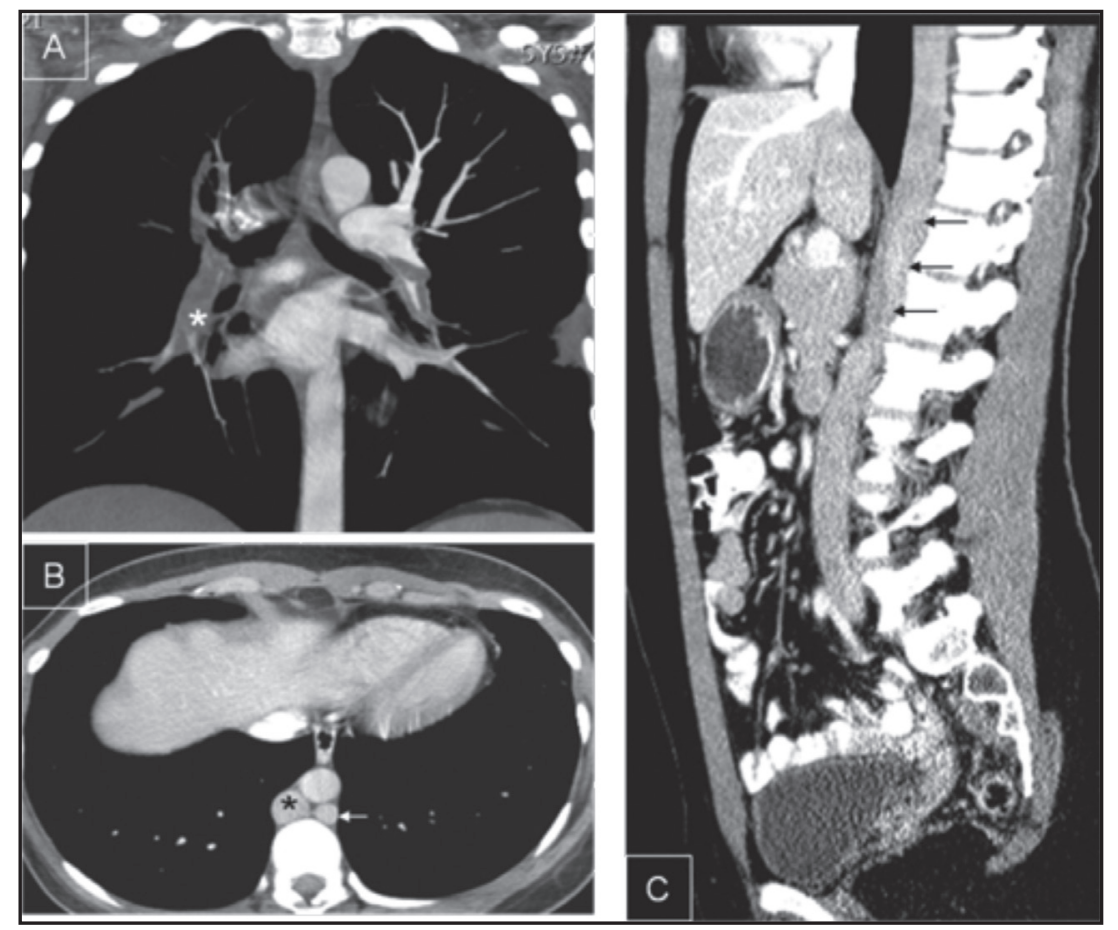

Figura 1. A. Angiotac de tórax, reconstrucción coronal: Trombos en la arteria interlobar (*) y arteria lobar superior derecha, trombos segmentarios en el lóbulo inferior izquierdo; B. Tomografía de abdomen, plano axial: aumento en el tamaño de la vena ácigos (*) y hemiácigos (flecha); C. Reconstrucción sagital: continuidad de la VCI al tórax como ácigos (flechas), con ausencia de su porción intrahepática, VCI suprahepática presente. 
Ambulatoriamente se realiza tamización de hipercoagulabilidad, la cual fue negativa (anticuerpos antinucleares, anticardiolipinas y anticoagulante lúpico, prueba de agregabilidad plaqueataria, homocisteína, mutación del factor $\mathrm{V}$ de Leyden, antitrombina III, proteína C, proteína S). Tras un año de uso de warfarina sin complicaciones, hematología y neumología consideran suspender la anticoagulación. El resultado del dímero $\mathrm{D}$ después de un mes de suspensión de la anticoagulación fue negativo. Se contraindica el uso de hormonas anticonceptivas y se dan instrucciones para evitar situaciones de riesgo trombótico. Ante la eventualidad de un nuevo evento trombótico tendría indicación de anticoagulación a largo plazo. Durante la observación de tres años la evolución de la paciente ha sido favorable sin eventos relevantes.

\section{Discusión}

Las diversas anomalías del desarrollo de la vena cava inferior son resultado de alteraciones durante su embriogénesis, que involucra la fusión y regresión de diferentes vasos embrionarios que dan lugar a la conformación definitiva de la misma, con sus segmentos intrahepático, suprarrenal, renal e infrarrenal (1).

La continuación de la vena cava inferior con la vena ácigos, también denominada ausencia del segmento hepático de la vena cava inferior, tiene una prevalencia de $0.6 \%$ (3). Ésta se produce por una falla en la anastomosis de la vena subcardinal y hepática, llevando a la atrofia de la vena subcardinal derecha, resultando en una vena cava inferior que entra al tórax como vena ácigos $(1,6)$ (Figura 2). Los individuos con esta malformación que no cursan con anomalías adicionales son generalmente asintomáticos y el diagnóstico es incidental (3).

Sin embargo, las malformaciones de la vena cava inferior pueden ser un factor de riesgo para el tromboembolismo venoso $(2,3)$ al predisponer a la estasis venosa aun ante la existencia de un sistema colateral compensatorio. En la población con trombosis venosa profunda, la prevalencia estimada de anormalidades de la vena cava inferior se ha descrito entre 5 y $9.5 \%$ (3), reportándose hasta en $16.2 \%$ de los pacientes con trombosis ilíaca en menores de 50 años (7). Existen casos reportados de malformaciones de la vena cava inferior asociados con tromboembolismo pulmonar aunque no se conoce la prevalencia real del diagnóstico en estos pacientes (3).

Múltiples tipos de malformaciones han sido documentadas asociadas a tromboembolismo venoso, entre ellas se han descrito la vena cava inferior ausente, hipoplasia o ausencia de alguno de sus segmentos y la duplicación de la vena cava inferior (8-10), las cuales pueden estar asociadas o no a otros factores de riesgo.

Se debe sospechar anomalía de la vena cava inferior en pacientes menores de 30 años con trombosis venosa profunda de miembros inferiores que se presenta de manera espontánea, especialmente cuando están involucradas las

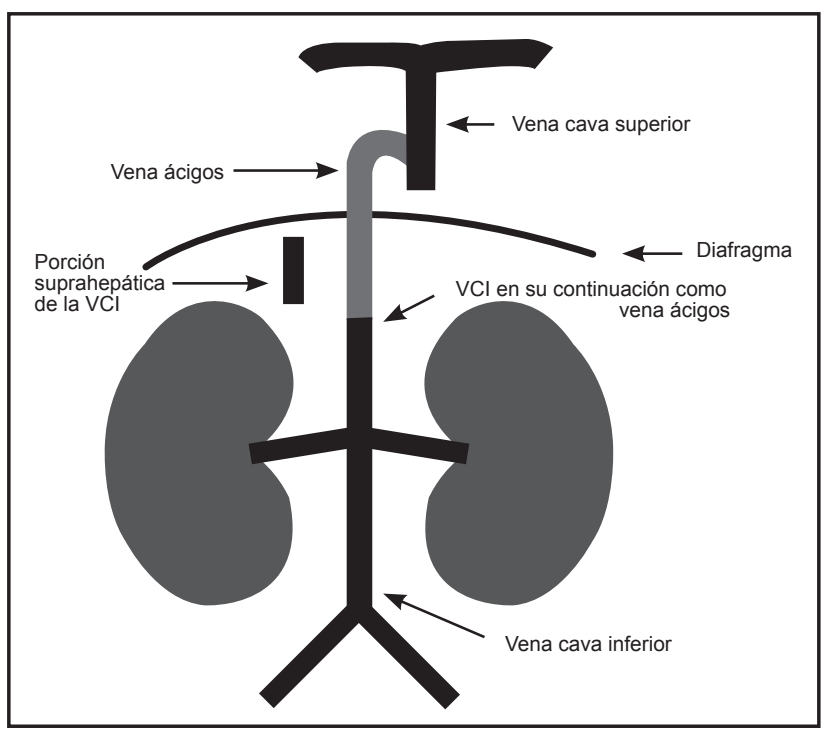

Figura 2. Esquema que muestra la continuación de la VCI con la vena ácigos, y la pre sencia por separado de la porción suprahepática de la VCI que finalmente desembocará en la aurícula derecha.

venas ilíacas, cuando hay resistencia a anticoagulantes, trombosis venosa profunda recurrente o presencia de trombosis venosa profunda bilateral, la cual puede presentarse hasta en $50 \%$ de los pacientes con malformaciones de la vena cava inferior, en comparación con menos de $10 \%$ de los pacientes con trombosis venosa profunda y sin malformación asociada $(2,7,9,10)$.

Para los casos de tromboembolismo pulmonar siempre que se encuentre una vena ácigos o hemiácigos dilatada en la tomografía de tórax se deben obtener imágenes adicionales para identificar anomalías de la vena cava inferior, como son tomografía abdominal o resonancia magnética (3).

Adicionalmente se deben tener en cuenta en los pacientes jóvenes otros factores de riesgo coexistentes y se debe considerar la tamización para trombofilias en los casos de un primer episodio de tromboembolismo venoso antes de los 40 años cuyo único factor de riesgo conocido sea la terapia con estrógenos o embarazo, como es el caso de nuestra paciente. La tamización para trombofilias no está indicada en todos los pacientes con un primer episodio de tromboembolismo venoso; ésta debe realizarse, aparte de las indicaciones ya mencionadas, en los pacientes menores de 60 años con un primer episodio no provocado de tromboembolismo venoso, pacientes con tromboembolismo venoso recurrentes aun en presencia de factores de riesgo, pacientes asintomáticos que sean familiares en primer grado de individuos con trombofilia sintomática demostrada, entre otros (11).

En cuanto a la duración de la anticoagulación no existe una recomendación clara específica para los pacientes con malformaciones de la vena cava. Se debe completar el tratamiento para tromboembolismo pulmonar y posteriormente la decisión de continuarlo a largo plazo debe ser individualizada en cada caso teniendo en cuenta la presencia de factores de riesgo adicionales inmodificables, número de 
eventos de tromboembolismo venoso presentados, resultado de dímero D posterior a la suspensión del tratamiento y el perfil de riesgo de sangrado, entre otros.

\section{Agradecimientos y financiación}

Agradecemos a la unidad de Neumología, Radiología y a la Clínica de Anticoagulación de la Fundación Santa Fe de Bogotá.

\section{Referencias}

1. Morató López AE, Quiróz Castro O, Ramírez Arias JL. Anomalías congénitas de la vena cava inferior, El rol de la tomografía computada multidetector. An Radiol Mex 2012; 11: 104-13.

2. Kreidy R, Salameh P, Waked M. Lower extremity venous thrombosis in patients younger than 50 years of age. Vasc Health Risk Manag. 2012; 8: 161-7.

3. Hyun DH, Seo JB, Chae EJ, Lee CW, Lee HJ, Kim M, et al. Pulmonary embolism associated with inferior vena cava interruption, Multidetector computed tomography findings. J Thorac Imaging. 2010; 25: W131-2.

4. Wells PS, Anderson DR, Rodger M, Ginsberg JS, Kearon C, Gent M, et al. Derivation of a simple clinical model to categorize patients probability of pulmonary embolism: increasing the models utility with the SimpliRED D-dimer. ThrombHaemost. 2000 Mar; 83(3): 416-20.
5. Aujesky D, Obrosky DS, Stone RA, Auble TE, Perrier A, Cornuz J. et al. Derivation and validation of a prognostic model for pulmonary embolism. Am J RespirCrit Care Med. 2005 Oct 15; 172(8): 1041-6.

6. Bass JE, Redwine MD, Kramer LA, Huynh PT, Harris JH Jr. Spectrum of Congenital Anomalies of the Inferior Vena Cava: Cross-sectional Imaging Findings. Radiographics. 2000; 20: 639-52

7. García-Fuster MJ, Forner MJ, Flor-Lorente B, Soler J, Campos S. Anomalías de la vena cava y trombosis venosa profunda. Rev Esp Cardiol. 2006; 59: 171-5.

8. Obernosterer A, Aschauer M, Schnedl W, Lipp RW. Anomalies of the inferior vena cava in patients with iliac venous thrombosis. Ann InternMed. 2002 Jan 1; 136(1): $37-41$.

9. Chee YL, Culligan DJ, Watson HG. Inferior vena cava malformation as a risk factor for deep venous thrombosis in the young. Br J Haematol. $2001 \mathrm{Sep}$; 114(4): 878-80.

10. Gayer G, Luboshitz J, Hertz M, Zissin R, Thaler M, Lubetsky A, et al. Congenital Anomalies of the Inferior Vena Cava Revealed on CT in Patients with DeepVein Thrombosis. AJR Am J Roentgenol. 2003 Mar; 180(3): 729-32.

11. Nicolaides A, Fareed J, Kakkar AK, Comerota AJ, Goldhaber SZ, Hull R, et al. Cardiovascular Disease Educational and Research Trust; European Venous Forum; North American Thrombosis Forum; International Union of Angiology and Union Internationale du Phlebologie. Thrombophilia. Clin Appl Thromb Hemost. 2013 Mar-Apr; 19(2): 177-87. 